\title{
Resonant Acoustic Mixing Method to Produce Lipid-based Liquid Crystal Nanoparticles
}

Dilek Yalcin ${ }^{a, b^{*}}$, Sarigama Rajesh c, Jacinta White a, Shaun C. Howard ${ }^{a}$,

Paul J. Pigram ${ }^{b}$, Nhiem Tran c, Benjamin W. Muir ${ }^{a^{*}}$

\section{a CSIRO Manufacturing, Clayton, Victoria 3168, Australia}

${ }^{\mathrm{b}}$ Centre for Materials and Surface Science, Department of Chemistry and Physics, School of Molecular Sciences, La Trobe University, Melbourne, Victoria 3086, Australia

'School of Science, RMIT University, Melbourne, Victoria 3000, Australia

\section{Supporting Information}

A
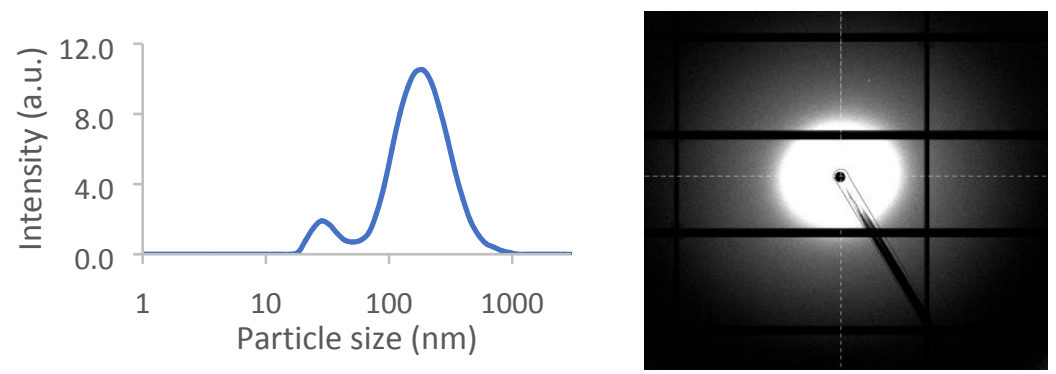

B
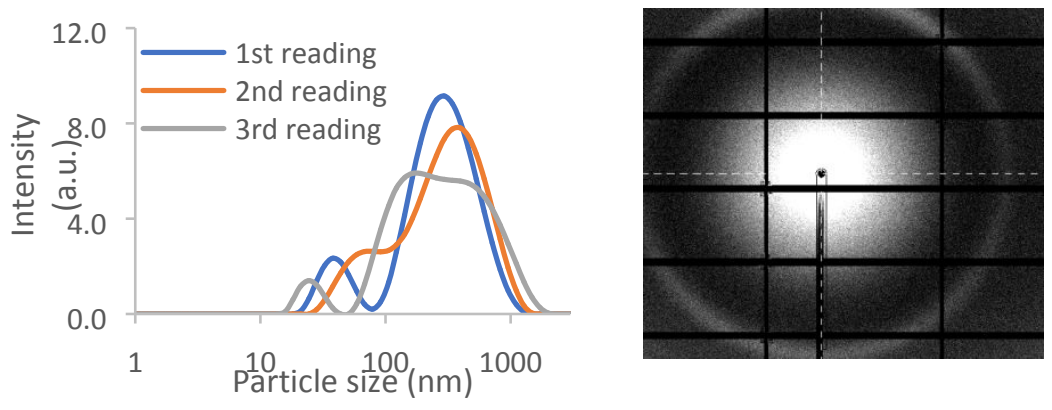

Figure S1. Size distribution and 2D SAXS images of PHYT-DDAB dispersions A. Sonication and B. RAM 
A
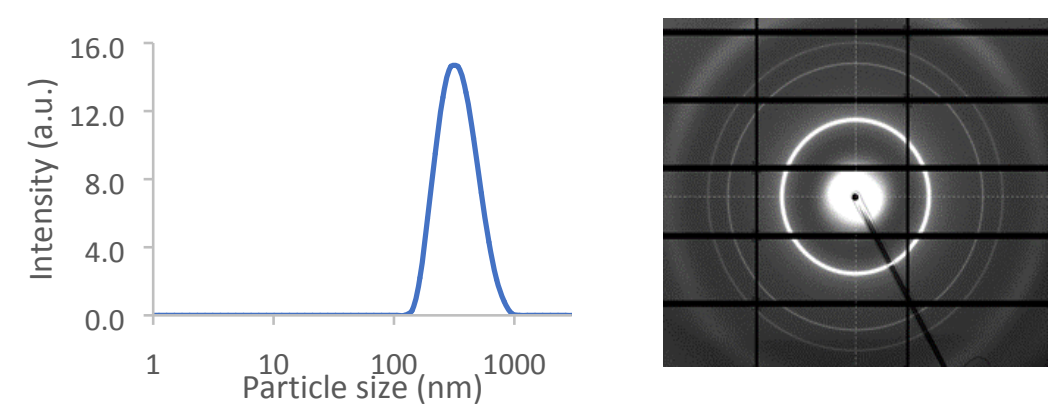

B
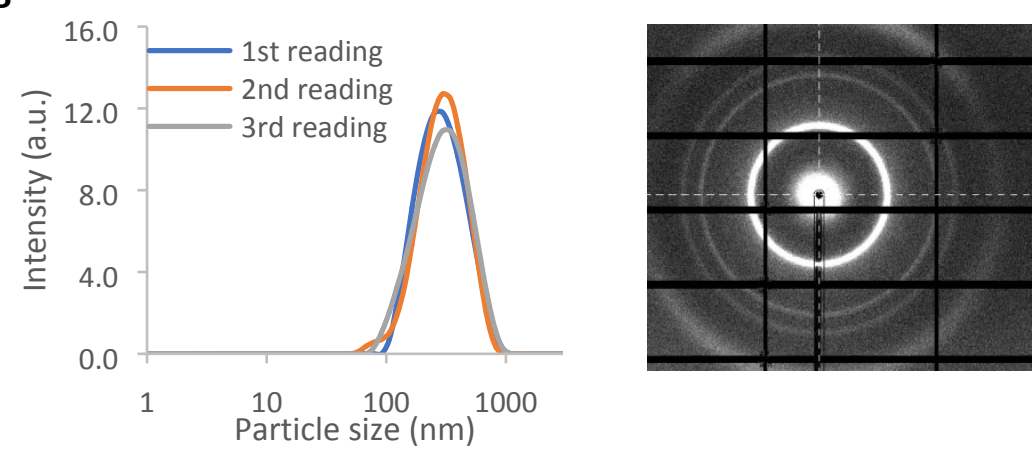

Figure S2. Size distribution and 2D SAXS images of MO-CA dispersions A. Sonication and B. RAM

A
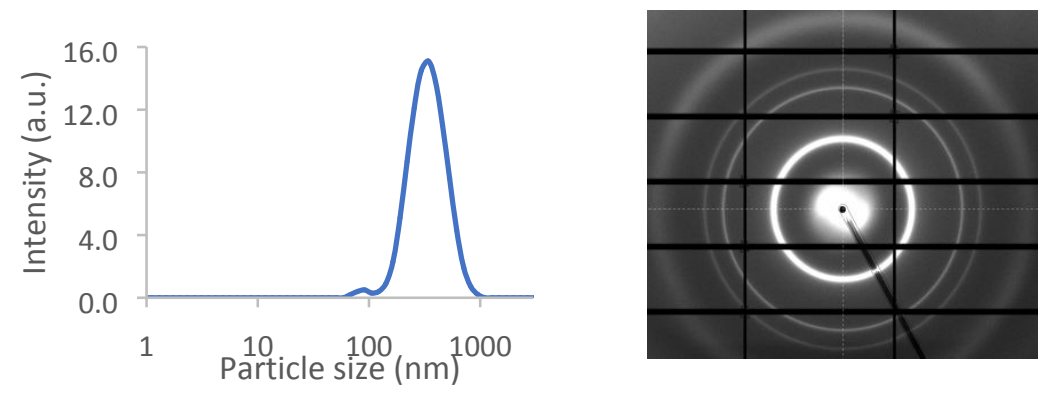

B
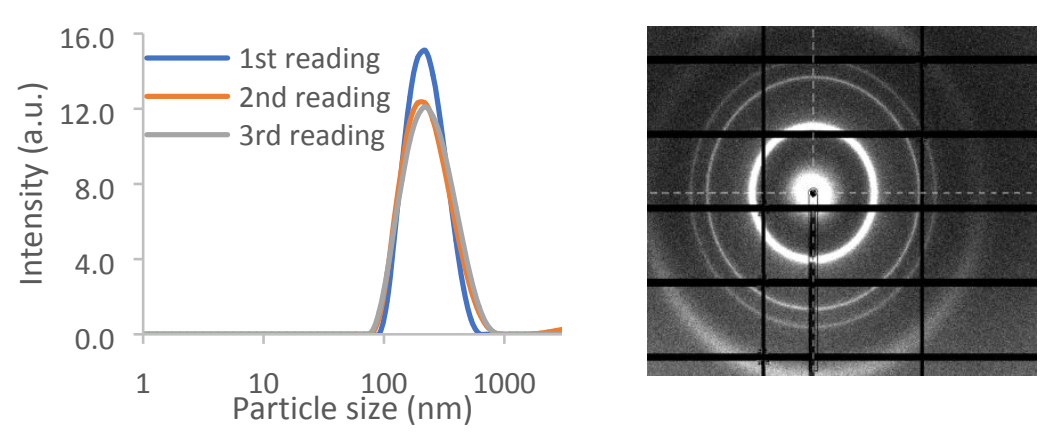

Figure S3. Size distribution and 2D SAXS images of binary mixture of PHYT-DDAB and MO-CA dispersions (1:1 by volume) A. Sonication and B. RAM 
A
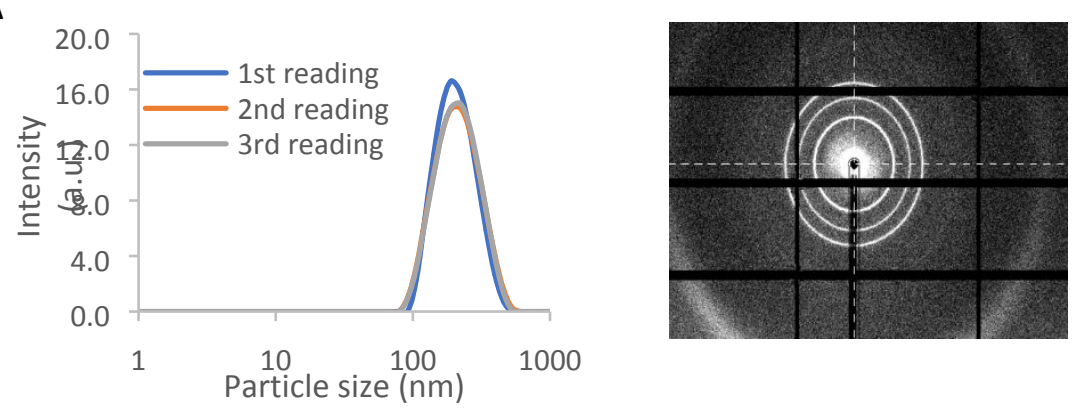

B
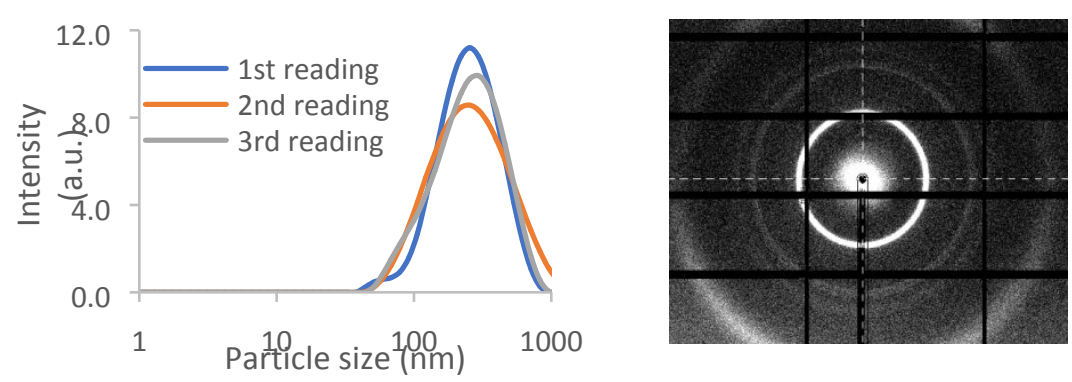

C
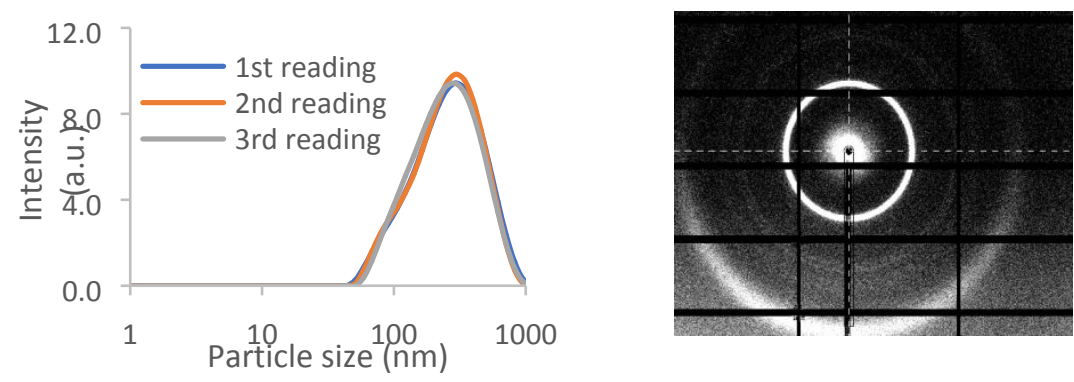

Figure S4. Size distribution and 2D SAXS images of nanoparticles fabricated by using RAM and mixed with PBS solution (1:2 by volume). A. PHYT-DDAB, B. MO-CA and C. Binary mixture of PHYT-DDAB and MO-CA dispersions (1:1 by volume) 
A
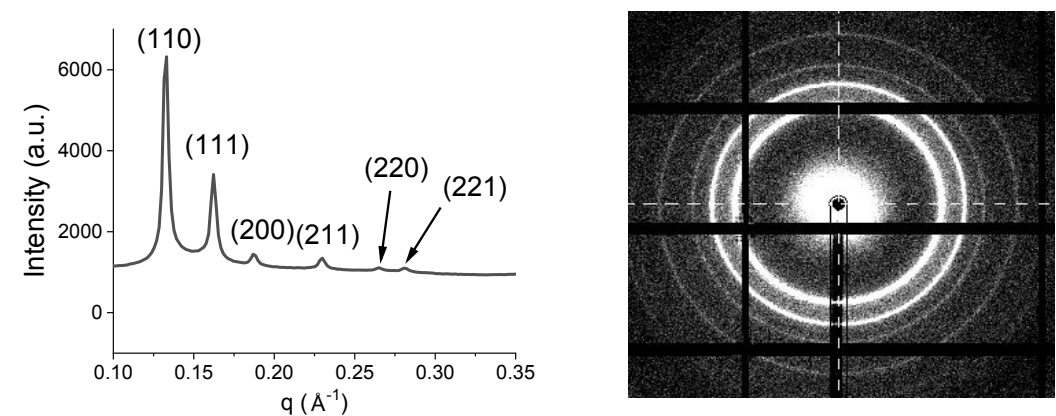

B
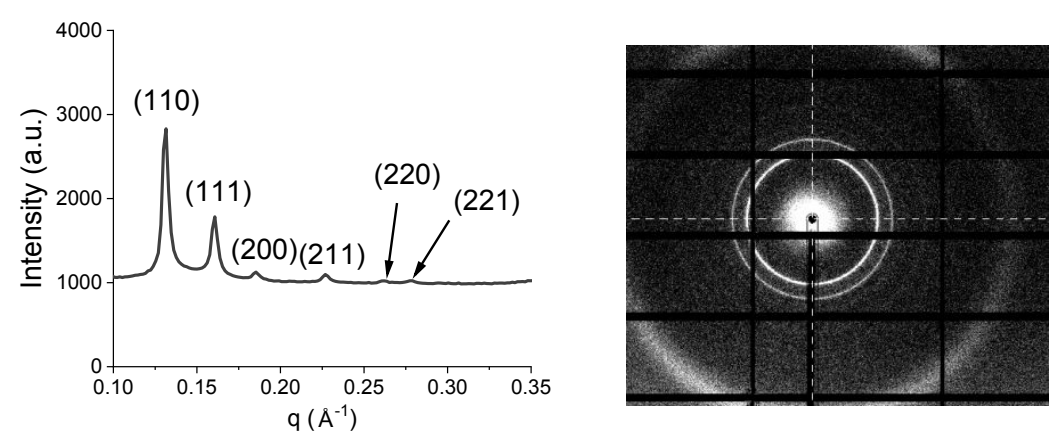

C
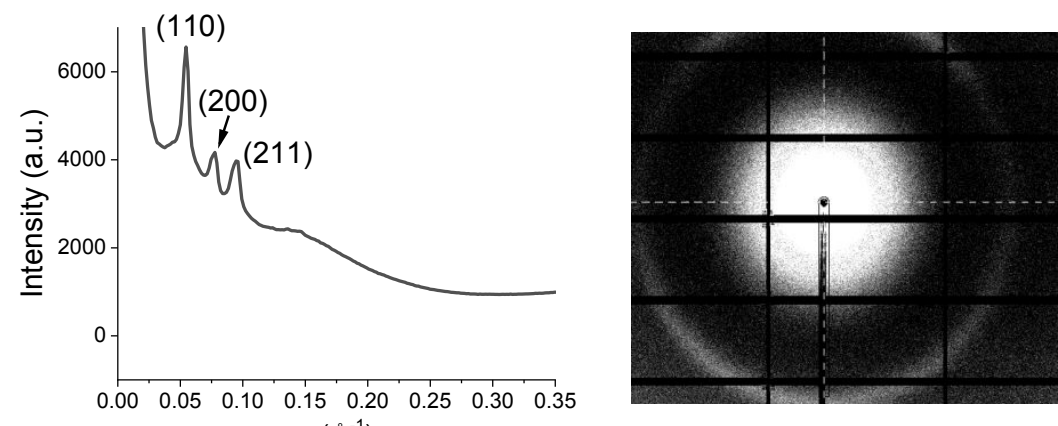

D
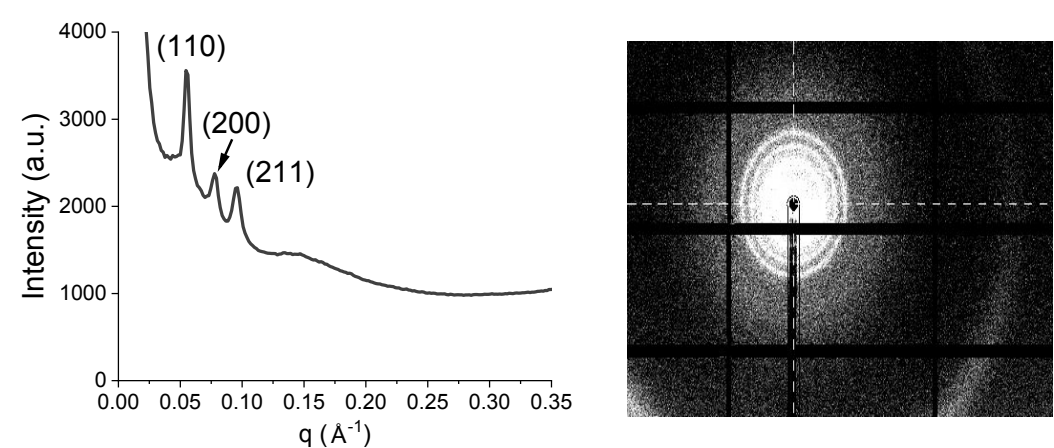

Figure S5. Scattering profiles and 2D SAXS images of samples A. PHYT dispersion in water, B. PHYT mixed with PBS solution (1:2 by volume), C. MO dispersion in water, and D. MO mixed with PBS solution (1:2 by volume). 


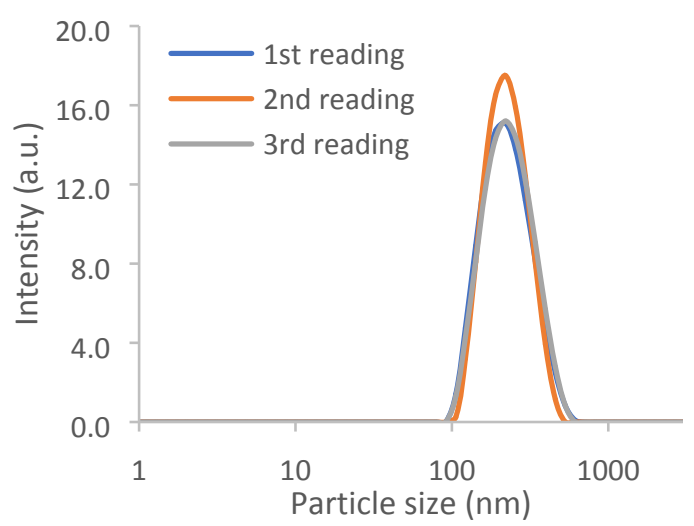

Figure S6. Size distribution curve of PHYT dispersion in water prepared in $5 \mathrm{~mL}$ container. 